OPEN ACCESS

Edited by:

Nuno Raimundo,

Universitätsmedizin Göttingen,

Germany

Reviewed by:

Giovanna Cenini,

University of Bonn, Germany

Luke Esposito,

Allen Institute for Brain Science,

United States

*Correspondence:

Serena Stanga

serena.stanga@uclouvain.be

Pascal Kienlen-Campard

pascal.kienlen-campard@uclouvain.be

${ }^{\dagger}$ Present Address:

Paolo E. Porporato,

Department of Molecular

Biotechnology and Health Sciences, Molecular Biotechnology Center,

University of Torino, Torino, Italy;

Matthew Bird,

Department of Clinical and Experimental Medicine, Hepatology, KU Leuven, Leuven, Belgium;

Claudia Marinangeli,

UMR-S 1172-JPArc-Centre de

Recherche Jean-Pierre AUBERT, CHU Lille, Institut National de la Santé et de la Recherche Médicale, Université de

Lille, Lille, France

${ }^{\ddagger}$ These authors have contributed equally to this work.

Specialty section:

This article was submitted to

Mitochondrial Research,

a section of the journal

Frontiers in Physiology

Received: 26 July 2017 Accepted: 28 September 2017 Published: 12 October 2017

Citation:

Contino $S$, Porporato $P E$, Bird $M$

Marinangeli C, Opsomer R,

Sonveaux P, Bontemps F, Dewachter I,

Octave J-N, Bertrand L, Stanga $S$ and

Kienlen-Campard P (2017) Presenilin

2-Dependent Maintenance of

Mitochondrial Oxidative Capacity and

Morphology. Front. Physiol. 8:796.

doi: 10.3389/fphys.2017.00796

\section{Presenilin 2-Dependent Maintenance of Mitochondrial Oxidative Capacity and Morphology}

\author{
Sabrina Contino ${ }^{1}$, Paolo E. Porporato ${ }^{2 \dagger}$, Matthew Bird ${ }^{1 \dagger}$, Claudia Marinangeli ${ }^{1+}$, \\ Rémi Opsomer ${ }^{1}$, Pierre Sonveaux ${ }^{2}$, Françoise Bontemps ${ }^{3}$, Ilse Dewachter ${ }^{1}$, \\ Jean-Noël Octave ${ }^{1}$, Luc Bertrand ${ }^{4}$, Serena Stanga ${ }^{1 * \neq}$ and Pascal Kienlen-Campard ${ }^{1 * \neq}$ \\ ${ }^{1}$ Alzheimer Research Group, Institute of Neuroscience, Université catholique de Louvain, Brussels, Belgium, \\ ${ }^{2}$ Pharmacology and Therapeutics, Institute of Experimental and Clinical Research, Université catholique de Louvain, \\ Brussels, Belgium, ${ }^{3}$ Metabolic Research Group, de Duve Institute, Université catholique de Louvain, Brussels, Belgium, \\ ${ }^{4}$ Pole of Cardiovascular Research, Institute of Experimental and clinical Research, Université catholique de Louvain, \\ Brussels, Belgium
}

Mitochondrial dysfunction plays a pivotal role in the progression of Alzheimer's disease (AD), and yet the mechanisms underlying the impairment of mitochondrial function in AD remain elusive. Recent evidence suggested a role for Presenilins (PS1 or PS2) in mitochondrial function. Mutations of PSs, the catalytic subunits of the $\gamma$-secretase complex, are responsible for the majority of inherited AD cases (FAD). PSs were shown to be present in mitochondria and particularly enriched in mitochondria-associated membranes (MAM), where PS2 is involved in the calcium shuttling between mitochondria and the endoplasmic reticulum (ER). We investigated the precise contribution of PS1 and PS2 to the bioenergetics of the cell and to mitochondrial morphology in cell lines derived from wild type (PS+/+), PS1/2 double knock-out (PSdKO), PS2KO and PS1KO embryos. Our results showed a significant impairment in the respiratory capacity of PSdKO and PS2KO cells with reduction of basal oxygen consumption, oxygen utilization dedicated to ATP production and spare respiratory capacity. In line with these functional defects, we found a decrease in the expression of subunits responsible for mitochondrial oxidative phosphorylation (OXPHOS) associated with an altered morphology of the mitochondrial cristae. This OXPHOS disruption was accompanied by a reduction of the $\mathrm{NAD}^{+} / \mathrm{NADH}$ ratio. Still, neither ADP/ATP ratio nor mitochondrial membrane potential $(\Delta \Psi)$ were affected, suggesting the existence of a compensatory mechanism for energetic balance. We observed indeed an increase in glycolytic flux in PSdKO and PS2KO cells. All these effects were truly dependent on PS2 since its stable re-expression in a PS2KO background led to a complete restoration of the parameters impaired in the absence of PS2. Our data clearly demonstrate here the crucial role of PS2 in mitochondrial function and cellular bioenergetics, pointing toward its peculiar role in the formation and integrity of the electron transport chain.

Keywords: presenilin, mitochondria, glycolysis, oxidative phosphorylation, cellular bioenergetics, Alzheimer's disease 


\section{INTRODUCTION}

Metabolic dysfunction is central in Alzheimer's disease (AD) since it appears at very early stage of the disorder, even before clinical symptoms (Chen and Zhong, 2013). It is evidenced in patients by a decrease in glucose utilization in temporoparietal association areas, together with cognitive decline and a severe failure in mitochondria oxidative metabolism (Herholz, 2012). Mitochondria are known as the powerhouse of the cell for their capacity to supply energy, but they are also critical in other cellular processes such as apoptosis, reactive oxygen species (ROS) production (Paradies et al., 2014), and calcium homeostasis (Osellame et al., 2012). All these processes turn out to be affected in AD pathology (Hroudova et al., 2014). Studies carried out either on AD patients' tissue samples or $\mathrm{AD}$ transgenic mice models reported an array of mitochondrial dysfunctions, including a morphological shift toward fission (Wang et al., 2009), a disrupted motility (Wang et al., 2009; Xie et al., 2013), an impairment of the electron transport chain (ETC) (Bosetti et al., 2002), and an increase in ROS production with deleterious effects on mitochondrial DNA integrity (Onyango et al., 2006). In cytoplasmic hybrid (cybrid) cell lines, generated by insertion of platelets' mitochondria collected from sporadic $\mathrm{AD}$ (SAD) patients into human neuroblastoma cells (SHSY5Y) depleted of mitochondria, bioenergetics dysfunctions such as oxidative phosphorylation (OXPHOS) and glucose utilization defects have been observed (Silva et al., 2013). These metabolic perturbations found in $\mathrm{AD}$ raised a chicken and the egg issue, namely to define whether mitochondrial dysfunction is a cause or a consequence in this pathology.

Recent studies suggested that Presenilins are involved in the control of mitochondrial functions (Behbahani et al., 2006; Filadi et al., 2016). PS1 and PS2 are two homologous polytopic aspartyl proteases identified as the catalytic subunits of the $\gamma$-secretase complex. PSs are directly involved in $\mathrm{AD}$ since the production of $A \beta$ is generated after the sequential cleavage of the Amyloid Precursor Protein (APP) by the $\beta$ and the $\gamma$-secretase (Zheng and Koo, 2011). A $\beta$ is the major component of senile plaques found in the brain of AD patients (Miller et al., 1993). Beside their key role in $\gamma$-secretase activity and $A \beta$ production, mounting evidence indicate additional roles for PSs in cell physiology, for which their catalytic activity seems less evident. PSs are involved in calcium homeostasis (Zhang et al., 2013), autophagy (Lee et al., 2010), neurotransmitters release (Zhang et al., 2009), synaptic plasticity and memory (Saura et al., 2004). Moreover, although PSs have different localization in the cell with PS1 mainly located at the plasma membrane and PS2 in the trans-Golgi network and

\footnotetext{
Abbreviations: $\Delta \Psi$, mitochondrial membrane potential; 1R1, PS1 knockout rescued PS1; 2R2, PS2 knockout rescued PS2; AD, Alzheimer disease; APP, amyloid precursor protein; CI-CV, complexes I-V; DTAB, dodecyltrimethylammonium bromide; ETC, electron transport chain; ER, endoplasmic reticulum; FAD, familial AD; FC, flow cytometry; FCCP, carbonyl cyanide-4-(trifluoromethoxy) phenylhydrazone; HPLC, high pressure liquid chromatography; MAM, mitochondria-associated membranes; MEF, mouse embryonic fibroblast; OCR, oxygen consumption rate; OXPHOS, oxidative phosphorylation; PS, presenilin; ROS, reactive oxygen species; SAD, sporadic AD; TEM, transmission electron microscopy; TMRM, tetramethylrhodamine methyl ester; WB, western blotting.
}

endosomal compartments (Meckler and Checler, 2016; Sannerud et al., 2016), they are both enriched in the mitochondriaassociated membranes (MAM) (Area-Gomez et al., 2009). MAM are specific membrane domains connecting endoplasmic reticulum (ER) and mitochondria. They are involved in lipid metabolism, calcium and cholesterol homeostasis (van Vliet et al., 2014; Filadi et al., 2017). PS2 has been reported to regulate the formation of ER/mitochondria contacts (Filadi et al., 2016) and calcium cross-talk between these two organelles (Zampese et al., 2011a). It is hence of particular interest to define (i) the precise contribution of PSs to the multiple aspects of mitochondrial function and (ii) to understand the respective contribution of PS1 or PS2 to these processes. Unraveling the role of PSs in cell metabolism is crucial to get insight in their physiological function and to understand how PS gain- or loss-of-function can create a pathological context, related for instance to $\mathrm{AD}$. We performed an array of metabolic measurements on Mouse Embryonic Fibroblasts cell lines (MEFs) derived either from wild type (PS+/+), PS1/2 double knock-out (PSdKO), PS2KO, and PS1KO mice embryos. We found a key role for PS2 (and not PS1) in cell metabolism and especially in OXPHOS and glycolysis. Indeed, the absence of PS2 altered the OXPHOS capacity and integrity but increased glycolytic flux to support energy needs. These pathways are crucial for energy-intensive consumer cells, like brain cells. Such defects might set the basis for further investigation of the metabolic impairments observed in $\mathrm{AD}$.

\section{MATERIALS AND METHODS \\ Cell Lines and Cell Culture}

Mouse Embryonic Fibroblasts cell lines (MEFs) derived from wild type $(\mathrm{PS}+/+), \mathrm{PS} 1 / 2$ double knock-out (PSdKO), PS2KO and PS1KO mice embryos were previously described (Hebert et al., 2006; Marinangeli et al., 2015). Rescued cell lines refer here to MEFs stably re-expressing human PS1 or PS2 in the corresponding single KO background: PS2KO rescued by human PS2 (2R2) and PS1KO rescued by human PS1 (1R1). Cells were maintained in DMEM low glucose $(5.5 \mathrm{mM}$ ) (Sigma-Aldrich, St Louis, USA) supplemented with penicillin/streptomycin solution (10 units-10 $\mu \mathrm{g}$ ) and 10\% fetal bovine serum (FBS) (Thermo Scientific, Rockford, USA). MEF 1R1 and 2R2 cell lines were generated by subcloning the human PSs cDNA sequences in the lentiviral backbone vectors plentiCMV/TOpuro and pTMtm898neo, respectively. Lentiviruses were produced in human embryonic kidney 293T cells. Supernatants containing the lentiviruses were concentrated with the Lenti-X Concentrator kit according to the manufacturer's instructions (Clontech Laboratories; California, USA). MEFs PS1KO and PS2KO were transduced by lentiviral particles followed by puromycin $(2.5 \mu \mathrm{g} / \mathrm{ml})$ selection (1R1) and G418 $(500 \mu \mathrm{g} / \mathrm{ml})$ selection (2R2), respectively. Stable expression of human PSs was monitored by western blotting (WB).

\section{Western Blotting}

WB was performed on cell lysates as previously described (Stanga et al., 2016). Primary antibodies used were Anti-OXPHOS 
Cocktail (1:1,000; Abcam, Cambridge, United Kingdom); AntiTOM20 (1:1,000; Proteintech, Rosemont, USA); anti-Presenilin (1:1,000; Cell Signaling, Danvers, USA); anti-Actin (1:3,000; Abcam, Cambridge, United Kingdom). Secondary antibodies used were anti-mouse (1:10,000; GE Healthcare, Little Chalfont, United Kingdom) and anti-rabbit (1:10,000; GE Healthcare, Little Chalfont, United Kingdom).

\section{Metabolic Measurements}

Oxygen consumption rate (OCR) measurements were performed with a Seahorse XF96 bioenergetic analyzer (Seahorse Bioscience; Massachusetts, USA). Cells were plated at $80 \%$ cell confluence onto a Seahorse 96 well plates $24 \mathrm{~h}$ before the assay. According to manufacturer's instructions, cell medium was replaced by the conditional medium (culture medium without FBS and sodium bicarbonate) and incubated at $37^{\circ} \mathrm{C}$ without $\mathrm{CO}_{2}$ for $1 \mathrm{~h}$ before completion of probe cartridge calibration. Inhibitors targeting the different mitochondrial complexes (Cell Mito Stress Test kit, Seahorse Bioscience) have been added sequentially to the cells during the experiment to measure the basal respiration, the coupling and the spare respiratory capacity: Oligomycin $(1 \mu \mathrm{M})$; FCCP $(1 \mu \mathrm{M})$; Rotenone and antimycin A $(0.5 \mu \mathrm{M})$. Results were normalized to the total amount of protein measured by the Bradford assay kit (Bio-Rad Laboratories, California, USA).

\section{Mitochondrial Membrane Potential $(\Delta \psi)$}

Fluorescent cationic probe tetramethylrhodamine methyl ester (TMRM) (Sigma-Aldrich, St Louis, USA) was used to evaluate the $\Delta \Psi$. The uncoupling agent Carbonyl cyanide-4(trifluoromethoxy) phenylhydrazone (FCCP) served as control (Sigma-Aldrich, St Louis, USA). Cyclosporin H ( $2 \mu \mathrm{M}$; Abcam, Cambridge, United Kingdom), an inhibitor of multidrug resistance pump activity was used to limit the efflux of TMRM. MEFs were incubated for $30 \mathrm{~min}$ at $37^{\circ} \mathrm{C}$ with TMRM $(30 \mathrm{nM})$, cyclosporine $\mathrm{H}$ with or without FCCP $(10 \mu \mathrm{M})$ diluted in KREBS medium. For the flow cytometry (FC) analysis we trypsinized cells seeded in 6 well plates and harvested them in PBS/5\% FBS. After centrifugation at $300 \mathrm{~g}$ for $5 \mathrm{~min}$, cells were resuspended in KREBS medium for the FC analysis performed on a BD FACSCanto $^{\text {TM }}$ flow cytometer (Biosciences; San Jose, USA). Data were analyzed with the FlowJo software (FlowJo,LLC; Oregon, USA).

\section{Adenine Nucleotides Analysis}

$24 \mathrm{~h}$ after seeding in $10 \mathrm{~cm}$ culture dishes, cells were harvested and lysed for $30 \mathrm{~min}$ on ice with $\mathrm{HCLO}_{4} 1 \mathrm{~N}$ (Sigma-Aldrich, St Louis, USA) and then centrifuged for $20 \mathrm{~min}$ at $10,000 \mathrm{~g}$ at $4^{\circ} \mathrm{C}$. The pellets were re-suspended in $\mathrm{NaOH} 0.5 \mathrm{M}$ for protein quantification and the supernatants were adjusted to $\mathrm{pH}$ 6-8 using a solution of $\mathrm{KOH} / \mathrm{KHCO}_{3} 3 \mathrm{M}$ (Sigma-Aldrich, St Louis, USA). Precipitated salts were separated from the liquid phase by centrifugation at $10,000 \mathrm{~g}$ at $4^{\circ} \mathrm{C}$ for $20 \mathrm{~min}$. Samples were stored at $-80^{\circ} \mathrm{C}$. Nucleotides were separated by high-performance liquid chromatography (HPLC) on $125 \times 4$ 4-mm PartiSphere 5 SAX anion-exchange column (Whatman, Maidstone, UK). Nucleotides were separated with a gradient from $100 \%$ buffer A $\left(0.01 \mathrm{M} \mathrm{NH}_{4} \mathrm{H}_{2} \mathrm{PO}_{4}, \mathrm{pH} 3.7\right)$ to $100 \%$ buffer $\mathrm{B}(0.48 \mathrm{M}$
$\mathrm{NH}_{4} \mathrm{H}_{2} \mathrm{PO}_{4}, \mathrm{pH} 3.7$ ) over $27 \mathrm{~min}$ at a flow rate of $2 \mathrm{ml} / \mathrm{min}$ according to the method of Hartwick and Brown (1975). UV detection of nucleotides was performed at $254 \mathrm{~nm}$. Quantification of nucleotides was achieved by peak integration of the area under the curve, validated by the use of external standards (ADP and ATP).

\section{NAD + /NADH Ratio}

Cells were seeded in 96 well plates at $80 \%$ confluence $24 \mathrm{~h}$ before the assay. $\mathrm{NAD}^{+} / \mathrm{NADH}$ ratio was measured by using the bioluminescent $\mathrm{NAD}^{+} / \mathrm{NADH}-\mathrm{Glo}^{\mathrm{TM}}$ assay kit (Promega, Wisconsin, USA) according to the manufacturer's instructions. Briefly, total $\mathrm{NAD}^{+}$and NADH were extracted from cell pellets with the basic solution $1 \%$ dodecyltrimethylammonium bromide (DTAB). Samples were divided in two for both acid and basic treatments and heated at $60^{\circ} \mathrm{C}$ for $15 \mathrm{~min}$. The oxidized form is selectively decomposed in the basic solution while the reduced form is decomposed in the acidic solution. For the luminescent reaction, samples were mixed with $100 \mu \mathrm{l}$ of $\mathrm{NAD}^{+} / \mathrm{NADH}-$ $\mathrm{Glo}^{\mathrm{TM}}$ detection reagent and incubated for $45 \mathrm{~min}$ before reading on the GloMax ${ }^{\circledR}$ 96-well plate luminometer (Promega, Wisconsin, USA).

\section{Glucose and Lactate Measurements}

Cells were seeded at $80 \%$ confluence in 6 well plates. $24 \mathrm{~h}$ after, $500 \mu \mathrm{l}$ of medium per well was collected and deproteinized. Glucose and lactate concentrations were measured using specific enzymatic assays on a CMA600 microdialysis analyzer (CMA Microdialysis AB, Solna, Sweden). Cells were collected for protein quantification and data were normalized to the amount of protein measured by BCA assay (Thermo Scientific, Rockford, USA).

\section{Glycolytic Flux Measurement}

Glycolytic rate was evaluated by measurement of the detritiation rate of $\left[3-{ }^{3} \mathrm{H}\right]$ glucose. Briefly, tritiated glucose $(0.2 \mu \mathrm{Ci} / \mathrm{ml}$; Perkin-Elmer; Massachussets, USA) was added to the medium (including $5.5 \mathrm{mM}$ glucose) for $30 \mathrm{~min}$. After medium removal, the tritiated water resulting from detritiated glucose was separated from the non-transported tritiated glucose by column chromatography and measured with the Tri Carb 2,810 liquid scintillation analyzer (Perkin Elmer; Massachussets, USA) as described previously (Marsin et al., 2002). Data were normalized to the amount of protein measured by BCA assay (Thermo Scientific, Rockford, USA).

\section{Complex I (Cl) Enzyme Activity Assay}

The activity of complex I of the mitochondrial respiratory chain was evaluated with the Complex I Enzyme Activity Dipstick Assay kit (Abcam, Cambridge, United Kingdom). Cells were scraped in PBS and centrifuged for $5 \mathrm{~min}$ at $4^{\circ} \mathrm{C}$ at $500 \mathrm{~g} .10$ volumes of extraction buffer were added to the pellets prior to 20 min of incubation on ice. After centrifugation, pellets were discarded and supernatants were used to determine protein concentration (BCA assay). Samples (corresponding to $30 \mu \mathrm{g}$ of proteins) were added to a microplate with blocking buffer. The dipsticks (containing an antibody capturing the CI) were 
immersed in the samples to capture the CI. The NADH and NBT were added to allow the oxidation of NADH by the complex I, which in turn reduce NBT to form a purple precipitate. Colorimetric signals were quantified with the Gel Doc 2,000 coupled with Quantity One software (Bio-Rad; California, USA).

\section{Transmission Electron Microscopy (TEM)}

Cells were fixed with $2.5 \%$ glutaraldehyde in phosphate buffer and kept in the fixative during $1 \mathrm{~h}$ at room temperature. They were washed and postfixed with $1 \%$ osmium tetroxide in the same buffer containing $0.8 \%$ potassium ferricyanide at $4^{\circ} \mathrm{C}$. The samples were dehydrated in acetone, infiltrated with Epon resin during 2 days, embedded in the same resin and polymerised at $60^{\circ} \mathrm{C}$ during $48 \mathrm{~h}$. Ultrathin sections were obtained using a Leica Ultracut UC6 ultramicrotome (Leica Microsystems, Vienna) and mounted on Formvar-coated copper grids. They were stained with $2 \%$ uranyl acetate in water and lead citrate. Sections were observed in a Tecnai Spirit electron microscope equipped with an Eagle CCD camera (FEI, Eindhoven, The Netherlands).

\section{Statistical Analysis}

The number of experiments for each experimental condition is indicated in the figure legends. Data were analyzed using GraphPad Prism software (GraphPad Software, La Jolla, CA, USA) by ANOVA followed by Bonferroni's multiple comparison tests. ${ }^{*} p<0.05,{ }^{* *} p<0.01,{ }^{* * * *} p<0.001$.

\section{RESULTS}

\section{Absence of PS2 Results in Decreased OXPHOS without Alteration of the $\Delta \Psi$}

PSs expression profile was measured by WB in MEF cell lines with antibodies directed against PS1 and PS2 CTFs (Figures 1A,B). The expression profile indicated the restoration of PSs expression in 2R2 (PS2 KO stably expressing human PS2) and 1R1 (PS1KO stably expressing human PS1). We evaluated the OXPHOS capacity by measurement of the OCR. The overall profile of OCR and the parameters related to the OXPHOS activity measured before or after drug addition, that are basal respiration, coupling (oxygen consumption devoted to ATP synthesis under resting conditions) and spare respiratory capacity (maximal uncoupled rate of respiration minus the basal rate) were all impaired in PSdKO and PS2KO cells (Figures 1C-F). Stable re-expression of PS2 in the PS2KO background (2R2) restored all these parameters to the levels measured in control cells (PS+/+). Importantly, the absence of PS1 did not affect mitochondrial respiration. We next measured the $\Delta \Psi$ with the TMRM probe by FC (Figure $2 \mathrm{~A}$ ) and by fluorescence measurement in microplates (Figure S1A). FC analyzes were performed on gated homogenous populations that accounted for more than $90 \%$ of the total population (Figure S1B). FCCP, an uncoupling agent abolishing $\Delta \Psi$ was used as a control. In both approaches (FC and direct fluorescence measurements), no significant differences in $\Delta \Psi$ were observed between the different populations of MEFs, although oxygen consumption was altered in PS2KO and PSdKO cells.

\section{Increased Anaerobic Glycolysis Sustains the Energy Production in PSdKO and PS2KO MEFs}

The observed PS2-dependent OXPHOS capacity decrease without impairment of $\Delta \Psi$ led us to evaluate the total ADP and ATP cellular levels. This was achieved by HPLC. No differences were observed in the ADP/ATP ratio between the cell lines (Figure 2B). Considering that we detected a significant decrease in coupling in PSdKO and PS2KO cells, we suspected that ATP production in PSdKO and PS2KO cells could result from an increased glycolysis (Figures 2C,D) that would counteract the defects observed in OXPHOS. We measured the levels of lactate secreted and glucose consumed by cells, as marker of glycolysis (Figure 2C). Both were indeed significantly higher in PSdKO and PS2KO cells. No changes were observed in PS1KO cells. Lactate secreted and glucose consumed measured in 2R2 cells were comparable to those measured in PS $+/+$ cells. These data were in line with the significant increase of the glycolytic flux (measured by detritiation of [3${ }^{3} \mathrm{H}$ ] glucose) observed in PSdKO and PS2KO cells, that was also restored in $2 \mathrm{R} 2$ to the levels measured in $\mathrm{PS}+/+$ cells (Figure 2D).

\section{Absence of PS2 Impairs the ETC}

So far, our results showed that mitochondrial oxidative capacity was impaired in the absence of PS2. This defect can be compensated by the increased glycolytic flux we observed. We further investigated the mitochondrial defects by profiling the expression of the five mitochondrial complexes. This was performed by WB with a cocktail of antibodies targeting representative subunits of the five mitochondrial complexes. Results showed that the expression of some complexes of the ETC was perturbed in PSdKO and PS2KO cells: CI and CII subunits were significantly decreased in both cell lines, and CIV was more specifically decreased in PS2KO cells. The expression profile of CI, CII, and CIV was restored in 2R2 cells (Figures 3A,B). To confirm these observations, we measured by an enzymatic assay the activity of the CI, which was significantly decreased in PSdKO and PS2KO cells but totally restored in 2R2 cells (Figure $3 \mathrm{C}$ ). Since CI uses $\mathrm{NADH}$ as an electron donor, we assessed whether the redox state was also affected by measuring the $\mathrm{NAD}^{+} / \mathrm{NADH}$ ratio. We measured the $\mathrm{NAD}^{+} / \mathrm{NADH}$ ratio using a luminescence assay. Consistent with the measurement of $\mathrm{CI}$ subunits by $\mathrm{WB}, \mathrm{NAD}^{+} / \mathrm{NADH}$ ratio was decreased in PSdKO and PS2KO cells and restored in 2R2 cells (Figure 3D).

\section{Mitochondrial Morphology in PSs-Deficient Cells}

We measured by WB in cell lysates the expression level of the mitochondrial import receptor subunit TOM20 (Figures 4A,B) as a marker of mitochondrial mass (Whitaker-Menezes et al., 2011). No significant difference in TOM20 levels was observed between the different cell types suggesting a comparable mass of mitochondria in all the cell lines tested, including PSdKO and PS2KO cells. Mitochondrial mass is 
A

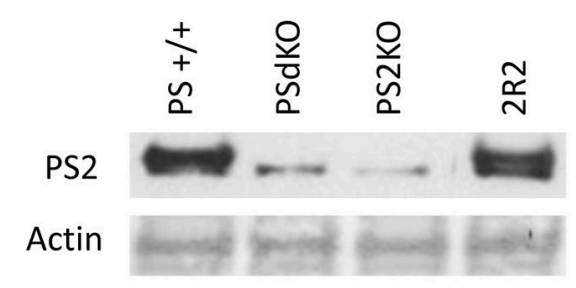

C

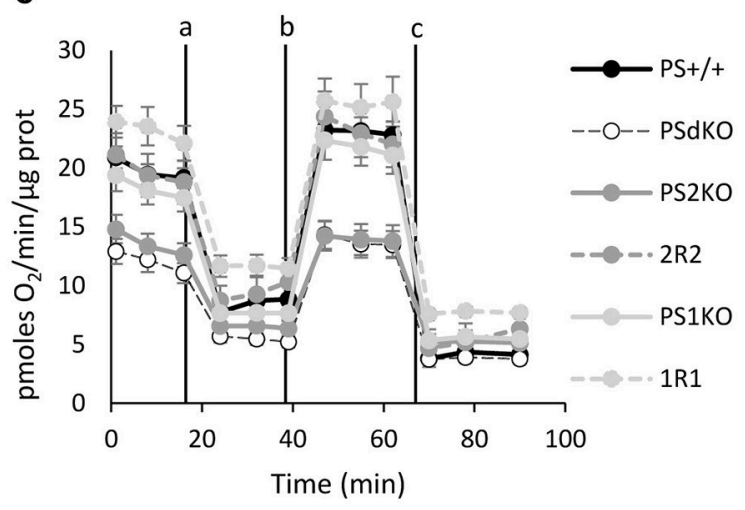

E

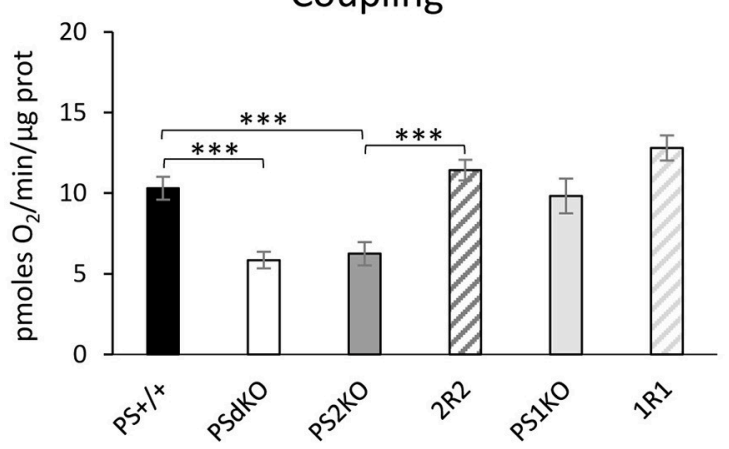

B

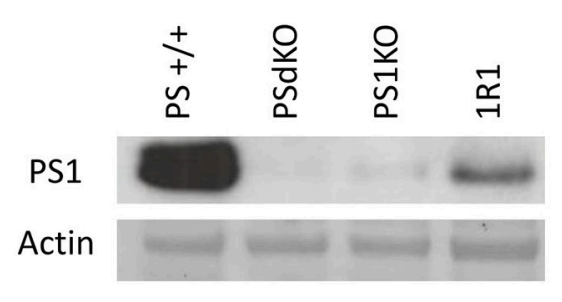

D

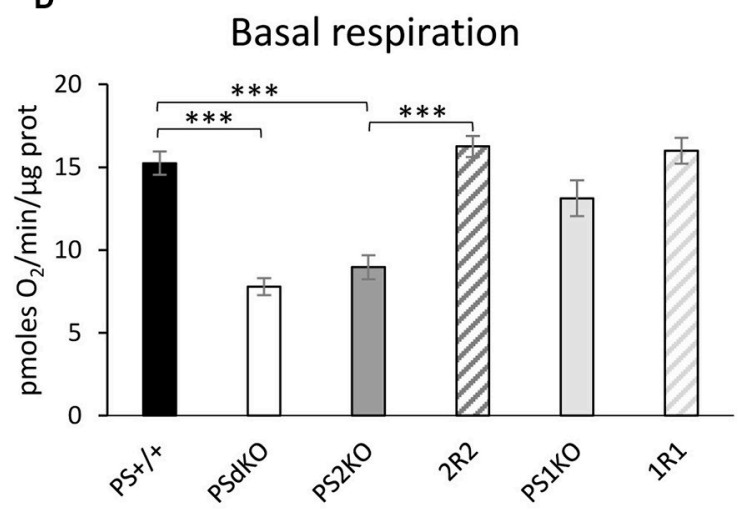

$\mathbf{F}$

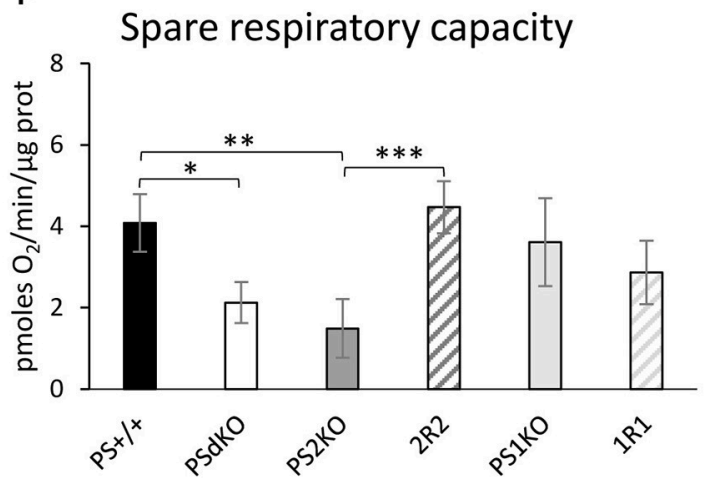

FIGURE 1 | Assessment of the OXPHOS capacity by measurement of the OCR. Experiments were carried out in MEF cell lines wild type (PS+/+), PS1/2 double KO (PSdKO), PS2KO, PS1KO and rescued cell expressing human PS1 and PS2 in PS1KO and PS2KO backgrounds, respectively (2R2 and 1R1). PSs expression (A,B) was analyzed by WB in cell lysates. Actin was used as a loading control. (C). OCR was determined using the Seahorse XF96 bioenergetic analyzer. Vertical lines indicate the time point at which the different compounds have been added: a. Oligomycin (CV inhibitor) b. FCCP $(\Delta \Psi$ uncoupler) C. Rotenone (Cl inhibitor) and antimycin A (CIII inhibitor). The basal respiration (D), the coupling ratio (E) and the spare respiratory capacity (F) were calculated according to the Cell Mito Stress Test kit's recommended protocol. Values (means \pm sem) are given in pmol $\mathrm{O}_{2} / \mathrm{min} / \mu \mathrm{g}$ protein. ${ }^{*} p<0.05,{ }^{* \star} p<0.01,{ }^{* * *} p<0.001(n=18$ from 3 independent experiments).

a gross indicator that could be only hardly related to the changes in mitochondrial function we observed. Thus, general mitochondrial morphology was evaluated by immunofluorescent staining targeting TOM20 (Figure S2), and no significant changes were observed about the shape and distribution of the mitochondrial network. Morphology and structure of single mitochondria were evaluated by TEM. Strikingly, we observed defective cristae (Figure 4C) in PSdKO and PS2KO cells when compared to PS $+/+$ cells. Cristae were less defined and less numerous in MEFs PSdKO and PS2KO and, importantly, these morphological defects were not observed in PS1KO cells.

\section{DISCUSSION}

The physiological function of the Presenilins 1 and 2 is far from being fully elucidated. Over the two last decades, the seminal observation identifying PS1 as the catalytic core of the $\gamma$-secretase (De Strooper et al., 1998; Kimberly et al., 2003) launched a considerable research effort aimed at understanding the exact role of PSs in $\gamma$-secretase activity and $A \beta$ production. However, an increasing number of studies focused on $\gamma$-secretase independent PSs functions such as regulation of calcium homeostasis (Zhang et al., 2013) or neurotransmitter (glutamate) release (Zhang et al., 2009). Since PSs were shown to be enriched 
A

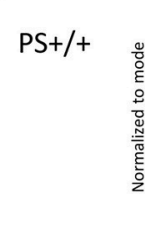

PSdKO
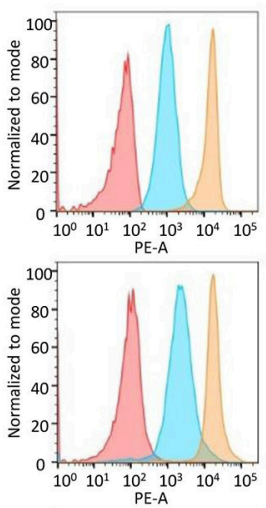

PS2KO

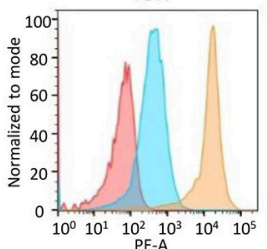

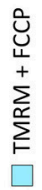

PS1KO

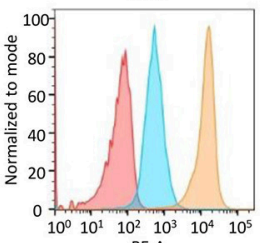

Merge
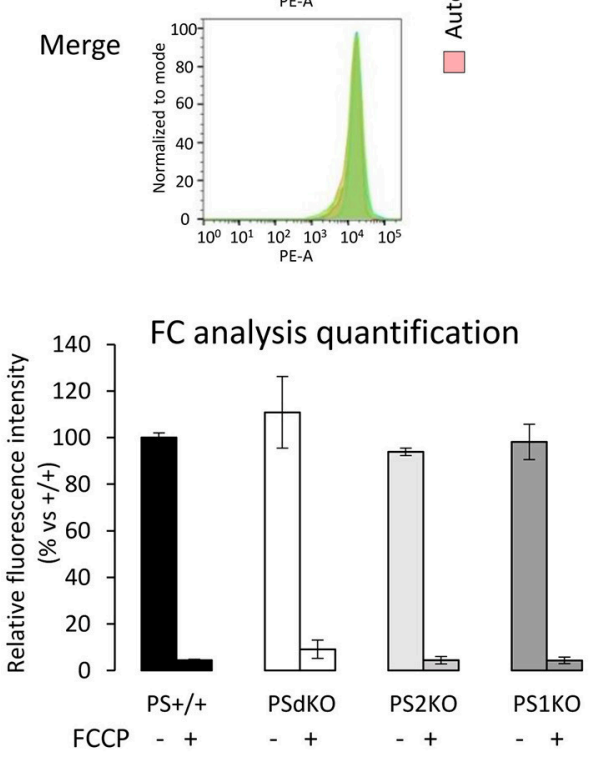

B

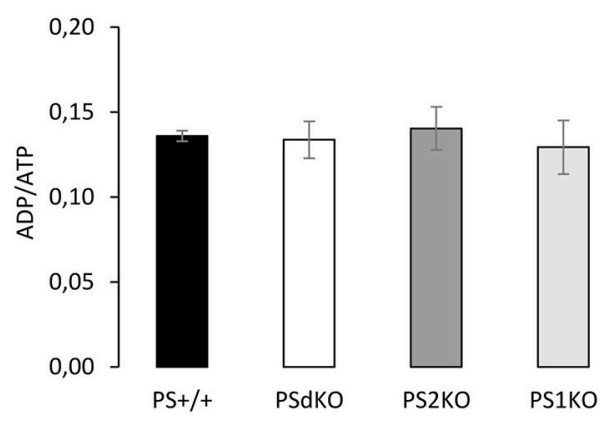

C

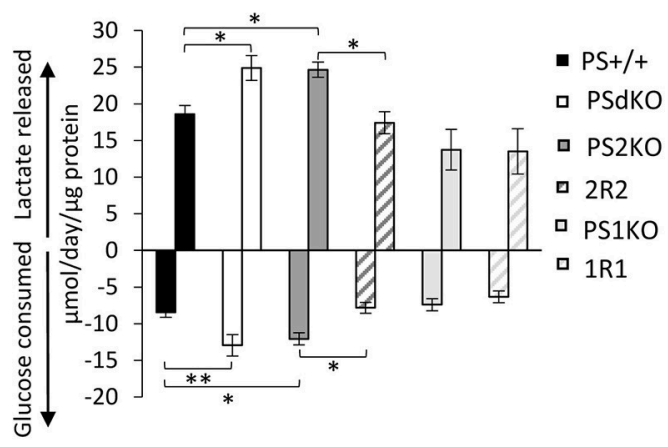

D

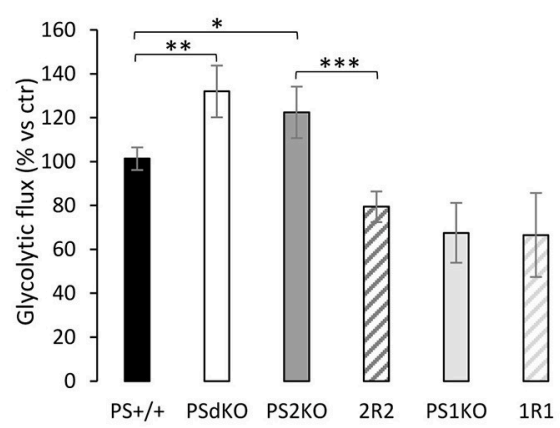

FIGURE 2 | Evaluation of the $\Delta \Psi$, ADP/ATP ratio and glycolysis. $\Delta \Psi$ was evaluated in MEF cells with the TMRM probe in the presence or absence of the $\Delta \Psi$ uncoupling agent FCCP and analyzed by FC ( $n=4$ from 2 independent experiments) (A). Histogram peaks represent (from left): autofluorescence; relative fluorescence of cells treated with TMRM+FCCP and of cells treated with TMRM only. The merge of histograms (in green) plots the overlapping signal obtained for the 4 cell lines in the TMRM only condition. Signals obtained were quantified (bottom) and results are expressed as the percentage of relative mean fluorescence intensity measured in PS+/+ cells. (B). ADP and ATP were measured by HPLC $24 \mathrm{~h}$ after seeding and data, expressed as ADP/ATP ratio, were normalized to protein content ( $n=6$ from 3 independent experiments). (C). Glucose consumption (negative columns) and lactate release (positive columns) were measured in supernatant from fresh medium after $24 \mathrm{~h}$ of culture. Data were normalized to protein content and expressed as $\mu$ mol/day $/ \mu \mathrm{g}$ prot. ${ }^{*} p<0.05,{ }^{* *} p<0.01$, $(n=16$ from 6 independent experiments). (D). Glycolysis rate was determined by the detritation rate of $\left[{ }^{3}-{ }^{3} \mathrm{H}\right]$ glucose after a 30 min incubation. Data were normalized to protein content. ${ }^{*} p<$ $0.05,{ }^{\star *} p<0.01,{ }^{* \star *} p<0.001$ ( $n=12$ from 6 independent experiments). 
A

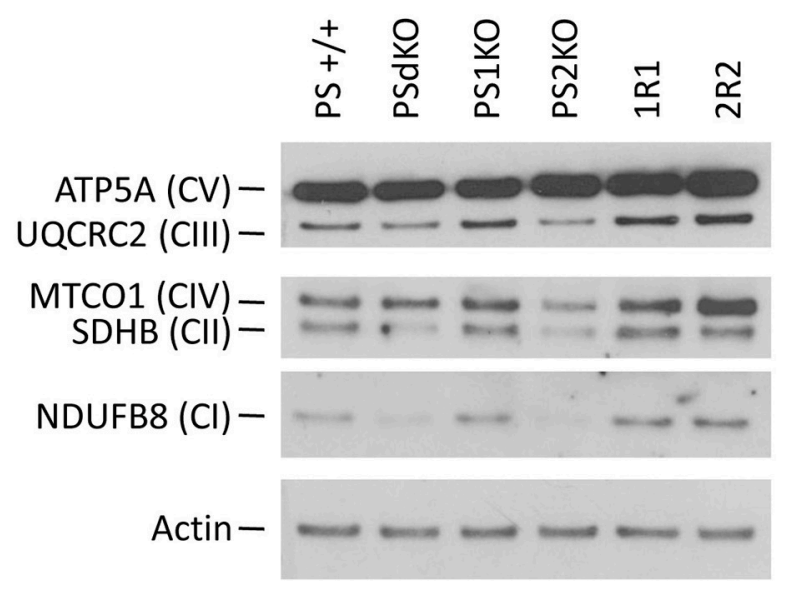

B

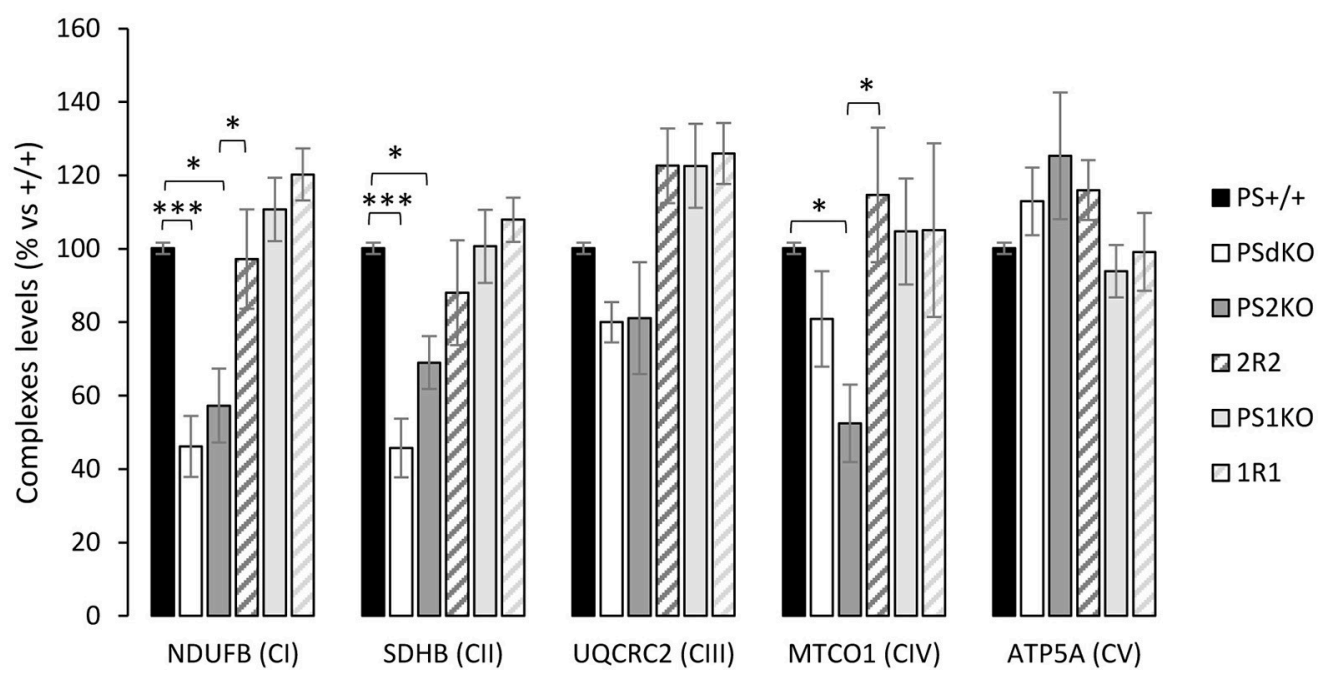

C

D
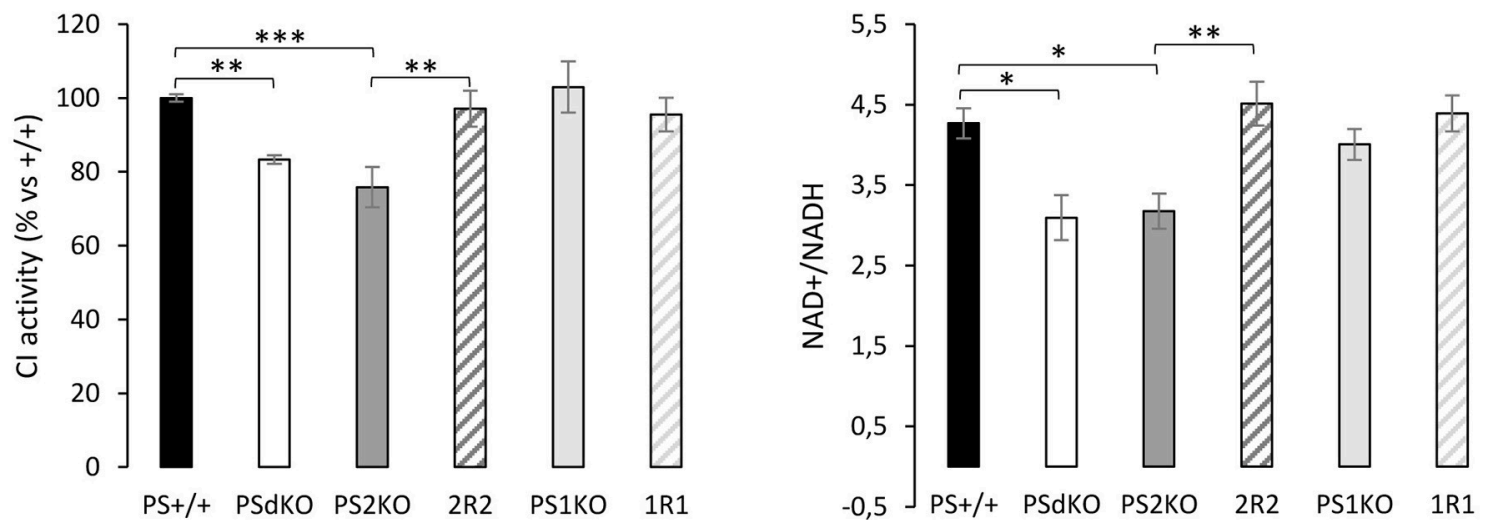

FIGURE 3 | Mitochondrial respiratory chain complexes expression profile, NAD ${ }^{+} / \mathrm{NADH}$ ratio and $\mathrm{Cl}$ activity. (A). The expression level of representative protein subunits from each of the five mitochondrial complexes (NDUFB8 for Cl; SDHB for Cll; UQCRC2 for Clll; MTCO1 for CIV; ATP5A for CV) was analyzed by WB on cell lysates. Actin was used as a loading control ( $n=5$ from 5 independent experiments). (B). WB quantifications (means \pm sem) are given as percentage of signal measured in PS $+/+$ cells. ${ }^{\star} p<0.05,{ }^{* \star *} p<0.001$. (C). Mitochondrial Cl activity was evaluated by the enzyme activity dipstick assay. ${ }^{* \star} p<0.01,{ }^{* \star *} p<0.001(n=12$ from 6 independent experiments). (D). NAD ${ }^{+} / \mathrm{NADH}$ ratio was quantified by a bioluminescent kit ${ }^{*} p<0.05,{ }^{* *} p<0.01$ ( $n=24$ from 8 independent experiments). 
A

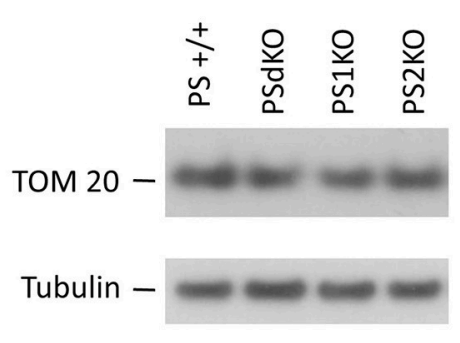

B

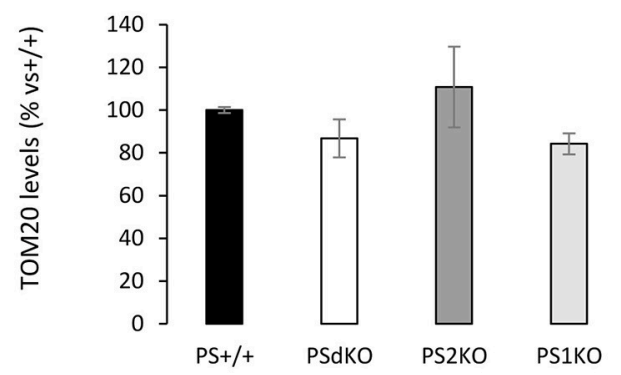

C
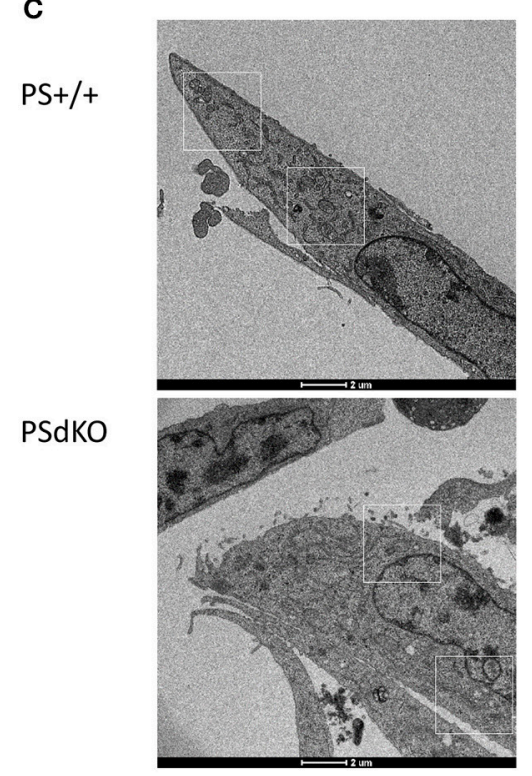

PS2KO

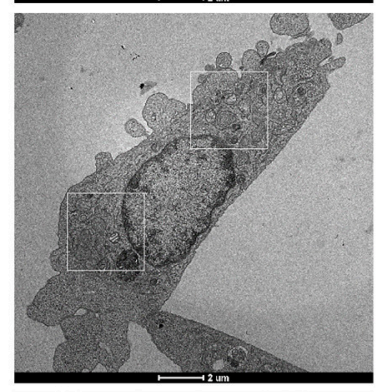

PS1KO

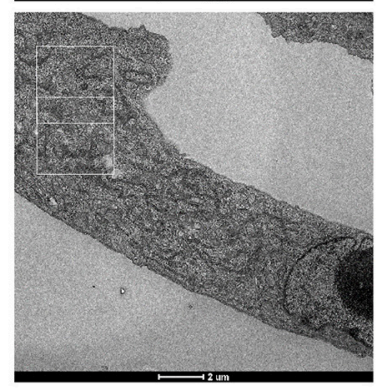

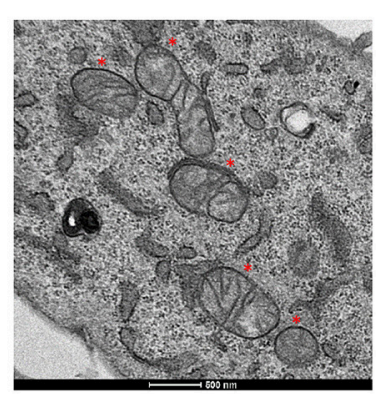
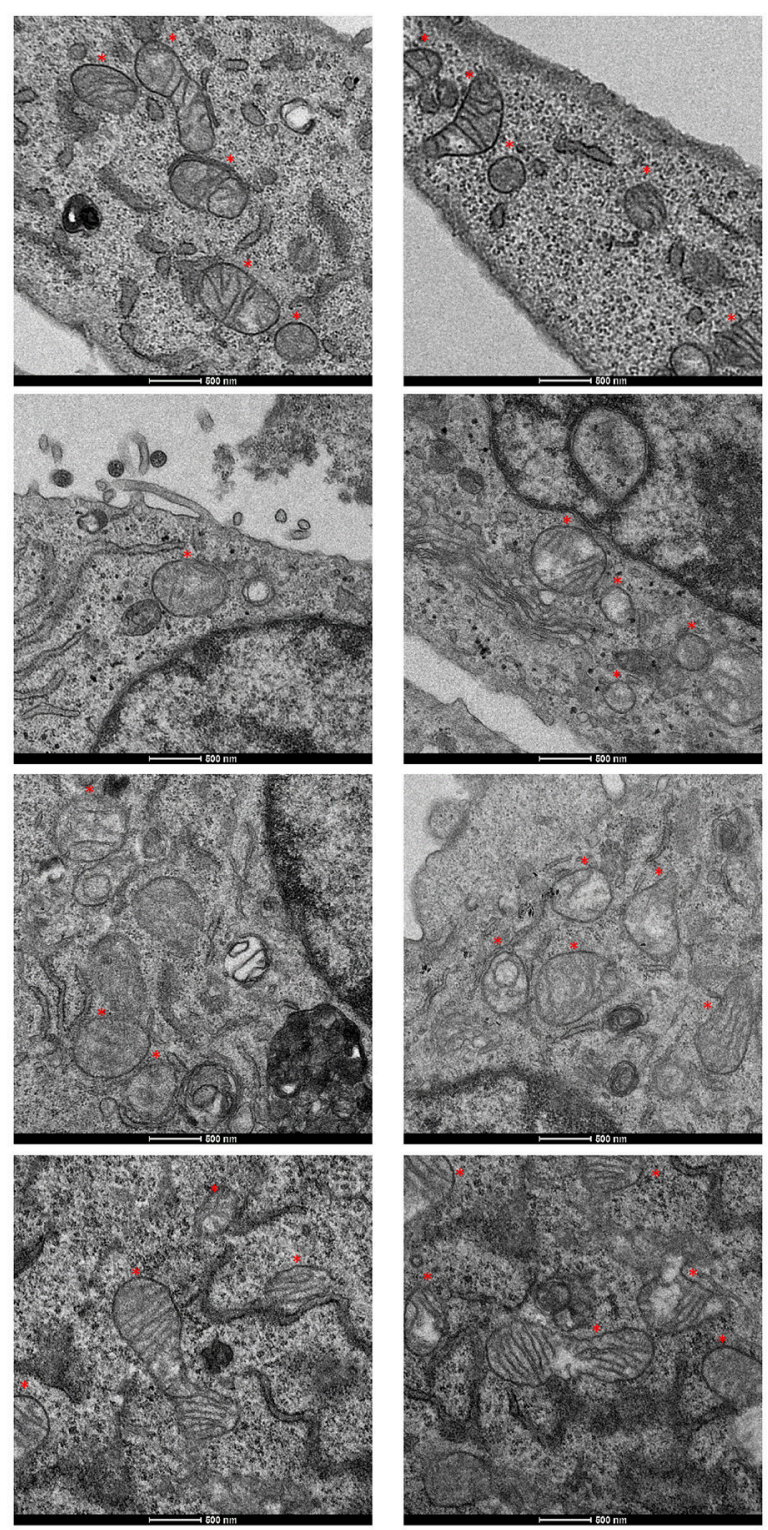

FIGURE 4 | Characterization of mitochondrial mass and morphology. TOM20 expression was analyzed by WB on cell lysates (A). Tubulin was used as a loading control. TOM2O quantification (B) (means \pm sem) is given as the percentage of the signal measured in the PS $+/+$ cells $(n=6$ independent experiments). (C) TEM's of cell section. Higher magnification regions (right columns) are boxed in white (left column). Red stars are indicating the position of the mitochondria on the micrograph. Scale bars are indicated on the bottom of the pictures. 
in MAM, a domain involved in different pathways related to ER and mitochondria functions (van Vliet et al., 2014; Filadi et al., 2017), we investigated the potential role of PSs in the control of cellular metabolism by measuring an array of parameters related to mitochondrial activity in PSs-deficient MEF cell lines. We observed in the absence of PS2, alterations in OXPHOS capacity and integrity associated with a decrease $\mathrm{NAD}^{+} / \mathrm{NADH}$ ratio. Along with these functional defects, the integrity of the mitochondrial cristae was also affected. These defects occurring in absence of PS2 were compensated by an increased glycolysis. Mitochondrial function was fully restored by stable re-expression of PS2 in our model.

\section{PS2 in OXPHOS and $\Delta \Psi$}

We measured the OCR as an indirect measurement of the OXPHOS. Our results indicate a significant decrease in PSdKO and PS2KO of the OCR. All the parameters we measured (basal respiration, coupling and spare respiratory capacity) were decreased in PSdKO and PS2KO cells, supporting a general defective OXPHOS capacity. One could have expected here an impact on the $\Delta \Psi$ and ATP production, since the ATP synthase uses the electrochemical gradient formed by pumping protons through the inner membrane to produce ATP. With the TMRM probe, we observed that the $\Delta \Psi$ measured is stable in all the cell types. Previous studies reported a $\Delta \Psi$ decrease in PSdKO and PS2 KO cells (Behbahani et al., 2006). This discrepancy could be due to different procedures as for instance the use of a different probe (JC-1) at high concentration (Perry et al., 2011). Behbahani et al. used like us FC analysis as a readout for $\Delta \Psi$, but it should be noted that in their case the use of the JC-1 probe to measure $\Delta \Psi$ changes implies a shift in the emission wavelength of the probe (with overlapping spectra) that renders the analysis by FC more difficult to interpret. With the TMRM probe, by using FC or direct fluorescent measurement in microplates (see Figure S1) as a readout we observed that the $\Delta \Psi$ is stable in all the cell types analyzed. $\Delta \Psi$ is also known to be one of the most stable parameter in the cell; even in the case of a deficit, the ATP synthase will work in reverse to keep it stable (Uechi et al., 2006).

To note, the main source of ROS production is the ETC, and their implication in $\mathrm{AD}$ could rise the question about their production in our model. We could expect a decrease of ROS production in PSdKO and PS2KO regarding of the OXPHOS deficit. A previous study showed a ROS increase in PSdKO compared to PS $+/+$, in serum deprivation conditions (Boo et al., 2009) that we do not modelize here. We performed few pilot ROS measurement (data not shown) in basal state but no significant message came up. It would be of interest, given the implication of ROS in $\mathrm{AD}$, to further study the PSs-dependent and ROS production in relevant models.

\section{PS2 and ATP Production}

Surprisingly, the ADP/ATP ratio we measured by HPLC was not impaired while the coupling (oxygen dedicated to ATP production) was significantly decreased in PSdKO and PS2KO cells. This is consistent with the increase of glycolytic flux and lactate production we observed in PSdKO and PS2KO cells. We used two distinct approaches to estimate glycolysis in our model: (i) we measured the levels of glucose consumed and lactate released in cell media; (ii) we measured the glycolysis rate by detritiation of $\left[3-{ }^{3} \mathrm{H}\right]$ glucose. These combined approaches showed that the glycolysis is increased significantly in PSdKO and PS2KO, providing the fuel to compensate their OXPHOS deficit. It should be noted that, similarly to undifferentiated stem cells (de Meester et al., 2014), MEF cell lines use anaerobic glycolysis even in the presence of oxygen to produce their energy. It would be of prime interest to analyze if such compensatory capacity exists in non-proliferative and oxidative cells like primary neurons. The metabolic phenotype resulting from altered PS2 function could be more drastic in neuronal cells. This would be very important in the context of the Alzheimer pathology, in which PSs play a central role. Zampese and collaborators highlighted in SH-SY5Y neuronal cells overexpressing a FAD PS2 mutant an enhanced $\mathrm{Ca}^{2+}$ transfer between ER and mitochondria (Zampese et al., 2011b). On the other hand, SH-SY5Y treated with siRNA specific for PS2 showed a decreased contact between mitochondria and $\mathrm{ER}$, which induced a decrease in calcium flux. The calcium crosstalk is known to be an important messenger for Krebs cycle enzymes stimulation and kinetics of the mitochondrial complexes. The mitochondrial calcium sequestration capacity is also important in the excitotoxicity and mitochondrial oxidative stress related to $\mathrm{AD}$. It is therefore possible that a lack of calcium flux could be one cause of the OXPHOS defect observed in our PS2KO cells.

\section{PS2 in ETC and Mitochondrial Network and Morphology}

We found that the bioenergetics defects observed were neither due to a decrease in the mitochondrial mass nor associated with a compensatory induction in mitochondrial mass. This favors the hypothesis of a direct disruption of the ETC activity in absence of PS2 rather than an overall defect in mitochondria biogenesis. This would be interesting to further address in postmitotic cells, such as neurons, where one might expect a proliferation of mitochondria as an attempt to compensate for the OXPHOS deficits. Such a compensatory induction was seen in heart and muscle (and to a lesser extent in brain) of mice deficient in the adenine nucleotide translocator-1 (ANT1), on which OXPHOS depends since it provides the ADP substrate (Esposito et al., 1999)

In order to understand the origin of the OXPHOS defect, we assessed the expression profile of the 5 mitochondrial complexes (CI-CV) of the ETC with a cocktail of antibodies. Even if we did not evaluate the expression of the entire complexes by this approach, we found the expression of several complexes of the ETC (CI, CII, and CIV) to be decreased in PSdKO and PS2KO cells. This was consistent with a functional analysis indicating a decreased CI activity in the same cells. The most important electron donor produced by the Krebs cycle and used by the $\mathrm{CI}$ is $\mathrm{NADH}$ and $\mathrm{NAD}^{+}$is an important co-factor regulating metabolic homeostasis (Canto et al., 2015). We therefore assessed their redox state by measuring the $\mathrm{NAD}^{+} / \mathrm{NADH}$ ratio and observed a significant decrease of the ratio in PSdKO and PS2KO cells. This can result either from an accumulation of $\mathrm{NADH}$ not used by the dysfunctional $\mathrm{CI}$ or from a decrease in $\mathrm{NAD}^{+}$ 
associated to metabolic pathways. $\mathrm{NAD}^{+}$and NADH could also both be affected and synergistically influence pathways regulating metabolism homeostasis (Canto et al., 2015).

The perturbation of the integrity of the ETC in PSdKO and PS2KO correlates with an altered structure of their cristae as evidenced by TEM. Cristae are the seat of the ETC, it is thus logical that the OXPHOS capacity and complexes expression are affected when their structure is altered. It has been demonstrated that cristae shape could regulate respiratory chain supercomplexes assembly and stability, impacting the respiratory efficiency (Cogliati et al., 2013). One of the key regulators of cristae shape is OPA1 and it would be interesting to further study the relationship between PS2 and OPA1. Alternatively, considering MAM as an important compartment for the homeostasis of lipids such as for example cardiolipin, one of the most prominent lipids in cristae (Paradies et al., 2014; Ikon and Ryan, 2017), we suggest that the morphological deficit of the cristae could be related to a dysregulation of MAM function in absence of PS2. To note, the MAM hypothesis has been proposed in $\mathrm{AD}$ since all the pathways (cholesterol, lipid and calcium homeostasis, mitochondrial OXPHOS stimulation) controlled by this compartment are altered in the pathology (Area-Gomez and Schon, 2017).

In conclusion, we reported here a specific physiological role of PS2 in cellular metabolism. Indeed, absence of PS2 results in defective mitochondrial cristae correlating with an impaired OXPHOS capacity and a modified redox state $\left(\mathrm{NAD}^{+} / \mathrm{NADH}\right.$ ratio). MEF cell lines, which are glycolytic cells, increase this glycolytic capacity to sustain their energy need in absence of PS2. This implies that impairment of these PS2-dependent processes could be involved in the progression of pathologies like $\mathrm{AD}$ in which PS2 play a key role. Investigation in an oxidative model and particularly in neurons are required to further explore this hypothesis.

\section{REFERENCES}

Area-Gomez, E., de Groof, A. J., Boldogh, I., Bird, T. D., Gibson, G. E., Koehler, C. M., et al. (2009). Presenilins are enriched in endoplasmic reticulum membranes associated with mitochondria. Am. J. Pathol. 175, 1810-1816. doi: 10.2353/ajpath.2009.090219

Area-Gomez, E., and Schon, E. A. (2017). On the Pathogenesis of Alzheimer's disease: the MAM Hypothesis. FASEB J. 31, 864-867. doi: 10.1096/fj. 201601309

Behbahani, H., Shabalina, I. G., Wiehager, B., Concha, H., Hultenby, K., Petrovic, N., et al. (2006). Differential role of Presenilin-1 and -2 on mitochondrial membrane potential and oxygen consumption in mouse embryonic fibroblasts. J. Neurosci. Res. 84, 891-902. doi: 10.1002/jnr.20990

Boo, J. H., Song, H., Kim, J. E., Kang, D. E., and Mook-Jung, I. (2009). Accumulation of phosphorylated beta-catenin enhances ROSinduced cell death in presenilin-deficient cells. PLoS ONE 4:e4172. doi: 10.1371/journal.pone.0004172

Bosetti, F., Brizzi, F., Barogi, S., Mancuso, M., Siciliano, G., Tendi, E. A., et al. (2002). Cytochrome $\mathrm{c}$ oxidase and mitochondrial F1F0-ATPase (ATP synthase) activities in platelets and brain from patients with Alzheimer's disease. Neurobiol. Aging 23, 371-376. doi: 10.1016/S0197-4580(01) 00314-1

\section{AUTHOR CONTRIBUTIONS}

SC, SS, and PK-C designed the research study; SC and SS conducted experiments with fundamental input of PEP, MB, and LB; all the authors analyzed data. SC, SS, and PK-C wrote the paper; all the authors have read and approved the final manuscript.

\section{FUNDING}

This work was supported by a grant of the Foundation for Research on Alzheimer's disease (PK-C), by the Interuniversity Attraction Pole Programme-Belgian Sate-Belgian Science Policy (IAP-P7/16 and IAP-P7/13) to J-NO, ID, and PK-C, by the Fondation Médicale Reine Elisabeth (FMRE) to J-NO, ID, and PK-C, by the Action de Recherche Concertée (ARC 14/19-059) to PK-C.

\section{ACKNOWLEDGMENTS}

We are grateful to Bart de Strooper (KU Leuven, Belgium) for the kind gift of MEF cell lines, to Bernadette Tasiaux (Alzheimer Research Group, UC Louvain, Belgium) for the helpful technical assistance, to Carmen López Iglesias for her kind assistance and to the Microscopy Core Lab of the Maastricht Multimodal Molecular Imaging Institute (M4I), FHML, University of Maastricht. LB; ID and PS are Senior Research Associates of Fonds National de la Recherche Scientifique (Belgium).

\section{SUPPLEMENTARY MATERIAL}

The Supplementary Material for this article can be found online at: https://www.frontiersin.org/articles/10.3389/fphys. 2017.00796/full\#supplementary-material

Canto, C., Menzies, K. J., and Auwerx, J. (2015). NAD(+) Metabolism and the control of energy homeostasis: a balancing act between mitochondria and the nucleus. Cell Metab. 22, 31-53. doi: 10.1016/j.cmet.2015.05.023

Chen, Z., and Zhong, C. (2013). Decoding Alzheimer's disease from perturbed cerebral glucose metabolism: implications for diagnostic and therapeutic strategies. Prog. Neurobiol. 108, 21-43. doi: 10.1016/j.pneurobio.2013.06.004

Cogliati, S., Frezza, C., Soriano, M. E., Varanita, T., Quintana-Cabrera, R., Corrado, M., et al. (2013). Mitochondrial cristae shape determines respiratory chain supercomplexes assembly and respiratory efficiency. Cell 155, 160-171. doi: 10.1016/j.cell.2013.08.032

de Meester, C., Timmermans, A. D., Balteau, M., Ginion, A., Roelants, V., Noppe, G., et al. (2014). Role of AMP-activated protein kinase in regulating hypoxic survival and proliferation of mesenchymal stem cells. Cardiovasc. Res. 101, 20-29. doi: 10.1093/cvr/cvt227

De Strooper, B., Saftig, P., Craessaerts, K., Vanderstichele, H., Guhde, G., Annaert, W., et al. (1998). Deficiency of presenilin-1 inhibits the normal cleavage of amyloid precursor protein. Nature 391, 387-390.

Esposito, L. A., Melov, S., Panov, A., Cottrell, B. A., and Wallace, D. C. (1999). Mitochondrial disease in mouse results in increased oxidative stress. Proc. Natl. Acad. Sci. U.S.A. 96, 4820-4825. doi: 10.1073/pnas.96.9.4820

Filadi, R., Greotti, E., Turacchio, G., Luini, A., Pozzan, T., and Pizzo, P. (2016). Presenilin 2 modulates endoplasmic reticulum-mitochondria coupling 
by tuning the antagonistic effect of mitofusin 2. Cell Rep. 15, 2226-2238. doi: 10.1016/j.celrep.2016.05.013

Filadi, R., Theurey, P., and Pizzo, P. (2017). The endoplasmic reticulummitochondria coupling in health and disease: molecules, functions and significance. Cell Calcium 62, 1-15. doi: 10.1016/j.ceca.2017.01.003

Hartwick, R. A., and Brown, P. R. (1975). The performance of microparticle chemically-bonded anion-exchange resins in the analysis of nucleotides. $J$. Chromatogr. 112, 650-662.

Hébert, S. S., Serneels, L., Tolia, A., Craessaerts, K., Derks, C., Filippov, M. A., et al. (2006). Regulated intramembrane proteolysis of amyloid precursor protein and regulation of expression of putative target genes. EMBO Rep. 7, 739-745. doi: $10.1038 /$ sj.embor.7400704

Herholz, K. (2012). Use of FDG PET as an imaging biomarker in clinical trials of Alzheimer's disease. Biomark. Med. 6, 431-439. doi: 10.2217/bmm.12.51

Hroudová, J., Singh, N., and Fišar, Z. (2014). Mitochondrial dysfunctions in neurodegenerative diseases: relevance to Alzheimer's disease. Biomed Res. Int. 2014:175062. doi: 10.1155/2014/175062

Ikon, N., and Ryan, R. O. (2017). Cardiolipin and mitochondrial cristae organization. Biochim. Biophys. Acta 1859, 1156-1163. doi: 10.1016/j.bbamem.2017.03.013

Kimberly, W. T., LaVoie, M. J., Ostaszewski, B. L., Ye, W., Wolfe, M. S., and Selkoe, D. J. (2003). Gamma-secretase is a membrane protein complex comprised of presenilin, nicastrin, Aph-1, and Pen-2. Proc. Natl. Acad. Sci. U.S.A. 100, 6382-6387. doi: 10.1073/pnas.1037392100

Lee, J. H., Yu, W. H., Kumar, A., Lee, S., Mohan, P. S., Peterhoff, C. M., et al. (2010). Lysosomal proteolysis and autophagy require presenilin 1 and are disrupted by Alzheimer-related PS1 mutations. Cell 141, 1146-1158. doi: 10.1016/j.cell.2010.05.008

Marinangeli, C., Tasiaux, B., Opsomer, R., Hage, S., Sodero, A. O., Dewachter, I., et al. (2015). Presenilin transmembrane domain 8 conserved AXXXAXXXG motifs are required for the activity of the gamma-secretase complex. J. Biol. Chem. 290, 7169-7184. doi: 10.1074/jbc.M114.601286

Marsin, A. S., Bouzin, C., Bertrand, L., and Hue, L. (2002). The stimulation of glycolysis by hypoxia in activated monocytes is mediated by AMP-activated protein kinase and inducible 6-phosphofructo-2-kinase. J. Biol. Chem. 277, 30778-30783. doi: 10.1074/jbc.M205213200

Meckler, X., and Checler, F. (2016). Presenilin 1 and presenilin 2 target gammasecretase complexes to distinct cellular compartments. J. Biol. Chem. 291, 12821-12837. doi: 10.1074/jbc.M115.708297

Miller, D. L., Papayannopoulos, I. A., Styles, J., Bobin, S. A., Lin, Y. Y., Biemann, K., et al. (1993). Peptide compositions of the cerebrovascular and senile plaque core amyloid deposits of Alzheimer's disease. Arch. Biochem. Biophys. 301, 41-52. doi: 10.1006/abbi.1993.1112

Onyango, I., Khan, S., Miller, B., Swerdlow, R., Trimmer, P., and Bennett, P. Jr. (2006). Mitochondrial genomic contribution to mitochondrial dysfunction in Alzheimer's disease. J. Alzheimers. Dis. 9, 183-193. doi: 10.3233/JAD-2006-9210

Osellame, L. D., Blacker, T. S., and Duchen, M. R. (2012). Cellular and molecular mechanisms of mitochondrial function. Best Pract. Res. Clin. Endocrinol. Metab. 26, 711-723. doi: 10.1016/j.beem.2012.05.003

Paradies, G., Paradies, V., De Benedictis, V., Ruggiero, F. M., and Petrosillo, G. (2014). Functional role of cardiolipin in mitochondrial bioenergetics. Biochim. Biophys. Acta 1837, 408-417. doi: 10.1016/j.bbabio.2013.10.006

Perry, S. W., Norman, J. P., Barbieri, J., Brown, E. B., and Gelbard, H. A. (2011). Mitochondrial membrane potential probes and the proton gradient: a practical usage guide. Biotechniques 50, 98-115. doi: 10.2144/000113610

Sannerud, R., Esselens, C., Ejsmont, P., Mattera, R., Rochin, L., Tharkeshwar, A. K., et al. (2016). Restricted Location of PSEN $2 / \gamma$-secretase determines substrate specificity and generates an intracellular A $\beta$ pool. Cell 166, 193-208. doi: 10.1016/j.cell.2016.05.020

Saura, C. A., Choi, S. Y., Beglopoulos, V., Malkani, S., Zhang, D., Shankaranarayana Rao, B. S., et al. (2004). Loss of presenilin function causesimpairments of memory and synaptic plasticity followed by age-dependent neurodegeneration Neuron 42, 23-36. doi: 10.1016/S0896-6273(04)00182-5

Silva, D. F., Selfridge, J. E., Lu, J., E, L., Roy, N., Hutfles, L., et al. (2013). Bioenergetic flux, mitochondrial mass and mitochondrial morphology dynamics in AD and MCI cybrid cell lines. Hum. Mol. Genet. 22, 3931-3946. doi: $10.1093 / \mathrm{hmg} / \mathrm{ddt} 247$

Stanga, S., Zanou, N., Audouard, E., Tasiaux, B., Contino, S., Vandermeulen, G., et al. (2016). APP-dependent glial cell line-derived neurotrophic factor gene expression drives neuromuscular junction formation. FASEB J. 30, 1696-1711. doi: 10.1096/fj.15-278739

Uechi, Y., Yoshioka, H., Morikawa, D., and Ohta, Y. (2006). Stability of membrane potential in heart mitochondria: single mitochondrion imaging. Biochem. Biophys. Res. Commun. 344, 1094-1101. doi: 10.1016/j.bbrc. 2006.03.233

van Vliet, A. R., Verfaillie, T., and Agostinis, P. (2014). New functions of mitochondria associated membranes in cellular signaling. Biochim. Biophys. Acta 1843, 2253-2262. doi: 10.1016/j.bbamcr. 2014.03.009

Wang, X., Su, B., Lee, H. G., Li, X., Perry, G., Smith, M. A., et al. (2009). Impaired balance of mitochondrial fission and fusion in Alzheimer's disease. J. Neurosci. 29, 9090-9103. doi: 10.1523/JNEUROSCI.135709.2009

Whitaker-Menezes, D., Martinez-Outschoorn, U. E., Flomenberg, N., Birbe, R. C., Witkiewicz, A. K., Howell, A., et al. (2011). Hyperactivation of oxidative mitochondrial metabolism in epithelial cancer cells in situ: visualizing the therapeutic effects of metformin in tumor tissue. Cell Cycle 10, 4047-4064. doi: $10.4161 / \mathrm{cc} .10 .23 .18151$

Xie, H., Guan, J., Borrelli, L. A., Xu, J., Serrano-Pozo, A., and Bacskai, B. J. (2013). Mitochondrial alterations near amyloid plaques in an Alzheimer's disease mouse model. J. Neurosci. 33, 17042-17051. doi: 10.1523/JNEUROSCI.1836-13.2013

Zampese, E., Fasolato, C., Kipanyula, M. J., Bortolozzi, M., Pozzan, T., and Pizzo, P. (2011a). Presenilin 2 modulates endoplasmic reticulum (ER)-mitochondria interactions and $\mathrm{Ca}^{2+}$ cross-talk. Proc. Natl. Acad. Sci. U.S.A. 108, 2777-2782. doi: $10.1073 /$ pnas. 1100735108

Zampese, E., Fasolato, C., Pozzan, T., and Pizzo, P. (2011b). Presenilin-2 modulation of ER-mitochondria interactions: FAD mutations, mechanisms and pathological consequences. Commun. Integr. Biol. 4, 357-360. doi: $10.4161 /$ cib.4.3.15160

Zhang, C., Wu, B., Beglopoulos, V., Wines-Samuelson, M., Zhang, D. Dragatsis, I., et al. (2009). Presenilins are essential for regulating neurotransmitter release. Nature 460, 632-636. doi: 10.1038/nature 08177

Zhang, S., Zhang, M., Cai, F., and Song, W. (2013). Biological function of Presenilin and its role in $\mathrm{AD}$ pathogenesis. Transl. Neurodegener. 2:15. doi: 10.1186/2047-9158-2-15

Zheng, H., and Koo, E. H. (2011). Biology and pathophysiology of the amyloid precursor protein. Mol. Neurodegener. 6:27. doi: 10.1186/1750-1326-6-27

Conflict of Interest Statement: The authors declare that the research was conducted in the absence of any commercial or financial relationships that could be construed as a potential conflict of interest.

Copyright (c) 2017 Contino, Porporato, Bird, Marinangeli, Opsomer, Sonveaux, Bontemps, Dewachter, Octave, Bertrand, Stanga and Kienlen-Campard. This is an open-access article distributed under the terms of the Creative Commons Attribution License (CC BY). The use, distribution or reproduction in other forums is permitted, provided the original author(s) or licensor are credited and that the original publication in this journal is cited, in accordance with accepted academic practice. No use, distribution or reproduction is permitted which does not comply with these terms. 